\title{
Factores de riesgo de estenosis de cuello vesical asociada a prostatectomía radical por carcinoma prostático - experiencia en un único centro
}

\section{Risk Factors of Bladder Neck Contracture after Radical Prostatectomy - A Single Institutional Experience}

\author{
Carlos Sánchez-Rodríguez ${ }^{1}$ Francisco Miguel González-Valverde ${ }^{2}$
}

Address for correspondence F. Miguel González Valverde, PhD, MD, Departamento de Cirugía, Facultad de Medicina de la Universidad de Murcia, Hospital General Universitario Reina Sofía de Murcia, Intendente Jorge Palacios 1, CP: 30.003, Murcia, España (e-mail: migova67@gmail.com).

Urol Colomb 2019;28:234-239.

\section{Resumen}

\section{Palabras Clave}

- neoplasia de próstata

- prostatectomia neocuello vesical
- estenosis de

Objetivo La estenosis de la anastomosis uretrovesical (EAU-V) afecta al 8,4\% de pacientes intervenidos de prostatectomía radical retropúbica por cáncer. Nuestro propósito fue estudiar los factores de riesgo de estenosis de cuello vesical asociada a prostatectomía radical por carcinoma prostático en nuestro centro.

Material y Métodos Estudio de casos-controles en una cohorte constituida por 120 pacientes intervenidos por adenocarcinoma prostático entre 2005 y 2012. Realizamos un análisis descriptivo de la muestra y el cálculo de la incidencia de EAU-V, un análisis univariante de los factores asociados mediante la t de Student y la chi cuadrado de Pearson, un análisis del tiempo transcurrido hasta la aparición de estenosis y una predicción del riesgo de EAU-V.

Resultados La incidencia de EAU-V tras prostatectomía radical en nuestro servicio fue del 22,5\%. Encontramos mayor riesgo en aquellos pacientes con débito por drenaje Jackson-Pratt (J-P) mayor de $500 \mathrm{cc}$, drenaje J-P retirado después del tercer día, estancia superior a 8 días, hemoglobina postoperatoria baja, PSA preoperatorio bajo, densidad de PSA elevada o que fueron intervenidos por el cirujano 1 o 5 . Todas las estenosis se produjeron en los 3 primeros años tras la cirugía. Los pacientes con un débito del drenaje por encima de $500 \mathrm{cc}$ y en los que el drenaje se retiró después del tercer día tienen 7,7 y 3,2 veces, respectivamente, más riesgo de padecer una EAU-V.

Conclusiones Nuestra incidencia de EAU-V y sus factores asociados coinciden con los publicados. El modelo predictivo desarrollado tiene utilidad meramente teórica ya que no abarca la totalidad de los factores que pueden influir en la aparición de la estenosis.

\section{Abstract}

Background Postprostatectomy vesicourethral anastomotic stenosis (VUAS) occurs in $8.4 \%$ of prostate cancer patients undergoing retropubic radical prostatectomy (RRP). received

April 4, 2017

accepted

November 6, 2017
DOI https://doi.org/ $10.1055 / \mathrm{s}-0038-1656519$ ISSN 0120-789X. eISSN 2027-0119.
Copyright $\odot$ 2019, Sociedad Colombiana License terms de Urología. Publicado por Thieme Revinter Publicações Ltda., Rio de Janeiro, Brazil. Todos los derechos reservados.

() (1) $\ominus \circledast$ 


\section{Keywords \\ - prostate neoplasms \\ - prostatectomy \\ - urinary bladder neck obstruction}

Our purpose was to study the risk factors of bladder neck contracture after radical prostatectomy in our center.

Materials and Methods Retrospective nested case-control study in a hospital-based cohort consisting of 120 patients underwent open or laparoscopic RRP from 2005 to 2012. We performed a descriptive analysis of the sample and calculated the incidence of postsurgical VUAS, a univariate analysis of factors associated with VUAS by Student $t$ test and Pearson chi-square test, an analysis of time to onset of stricture and a prediction of risk of stricture in patients undergoing RRP.

Results The incidence of VUAS after radical prostatectomy in our department was $22.5 \%$. We found a higher risk in patients in which surgical drainage was over $500 \mathrm{cc}$, the Jackson-Pratt drain was removed after the third day, length of stay $>8$ days, a low postoperative haemoglobin, a low preoperative PSA, an elevated PSA density and/or were operated on by surgeon 1 or 5 . All strictures occurred within the first 3 years after surgery. We predicted that patients with total drainage volume over $500 \mathrm{cc}$ and removal day after the third day would be 7.72 and 3.21 times more likely to have VUAS, respectively.

Conclusions Data from VUAS incidence and its associated factors are similar to those found in the literature. The developed predictive model has only a theoretical utility, because it does not cover all the influencing factors of the onset of the stricture.

\section{Introducción}

La estenosis de la anastomosis uretrovesical (EAU-V) sucede en un $0,4-32 \%$ de los pacientes intervenidos de prostatectomía radical (PR $)^{1}$ por cáncer de próstata, con una incidencia media de $8,4 \%{ }^{2}$ Generalmente, es el resultado de la formación de tejido cicatricial que cierra y reduce la configuración normal del cuello vesical. Ese estrechamiento provoca un aumento de la frecuencia y de la urgencia urinaria, chorro débil, vaciado incompleto de la vejiga e incluso retención aguda de orina. ${ }^{3}$

Múltiples factores de riesgo contribuyen al desarrollo de la EAU-V, incluidos los factores relacionados con el paciente y consideraciones técnicas en el momento de la cirugía. Se han propuesto factores preoperatorios como las características demográficas (edad, raza,..), comorbilidades, el PSA y el Gleason preoperatorios, el volumen prostático o los antecedentes de resección transuretral de próstata. También parecen existir condicionantes técnicos intraoperatorios (la eversión de la mucosa, pérdida de sangre intraoperatoria, tipo de sutura, preservación del paquete neurovascular), perioperatorios (prolongación del mantenimiento del drenaje quirúrgico y de la sonda Foley vesical) y postoperatorios (Gleason postoperatorio, incontinencia e infecciones del tracto urinario). No obstante, el peso de cada uno de esos factores en la aparición de la EAU-V, es objeto de controversia. El tratamiento inicial comienza con terapias endoscópicas, incluyendo la dilatación, la incisión transuretral (TUIBNC), y la inyección de agentes adyuvantes. En casos seleccionados, cuando la EAU-V sigue siendo refractaria a esas terapias, la reconstrucción quirúrgica de la anastomosis vesicouretral o derivación urinaria es una opción a tener en cuenta. ${ }^{3}$
Nuestro propósito ha sido estudiar los factores de riesgo de estenosis de cuello vesical asociada a prostatectomía radical retropúbica por carcinoma prostático en nuestro centro y comparar los hallazgos con los referidos estudios realizados en otros entornos hospitalarios.

\section{Material y Métodos}

Se ha realizado un estudio retrospectivo de casos y controles dentro de una cohorte ("nested case-control studies"), de base hospitalaria constituida por 120 pacientes intervenidos en nuestro hospital por adenocarcinoma de próstata. La cohorte está compuesta por todos los pacientes intervenidos de adenocarcinoma de próstata, mediante prostatectomía abierta según técnica de Walsh o laparoscópica en el Servicio de Urología del Hospital General Universitario Reina Sofía de Murcia, entre el 1 de octubre de 2005 y el 1 de agosto de 2012. Se consideraron como casos aquellos sujetos que desarrollaron EAU-V postoperatoria, y como controles al grupo de pacientes que no desarrollaron esa complicación. La totalidad de los sujetos se encuentran en control hasta el 1 de febrero de 2017 mediante seguimiento en consulta, con registro de las complicaciones postoperatorias. La información se obtuvo de la base de datos del Servicio de Documentación, del visor de imágenes Risweblink 1.5.7. (Siemens@, Hamburgo, Alemania) y de las historias clínicas en formato papel de cada paciente. Los datos fueron tratados mediante el programa SPSS 19 para Windows (IBM ${ }^{\circledR}$ SPSS Statistics Inc., Chicago IL. EEUU).

Se calcularon las frecuencias y los porcentajes para las variables cualitativas, y las medias, desviaciones estándar y valores máximos y mínimos para las mediciones cuantitativas. Realizamos el cálculo de la incidencia de EAU-V posquirúrgica, un análisis univariante de los factores asociados a ella 
mediante la prueba t de Student, la prueba chi cuadrado de Pearson, un análisis del tiempo transcurrido hasta la aparición de la estenosis, y una predicción del riesgo de estenosis en pacientes intervenidos mediante prostatectomía radical.

\section{Resultados}

\section{Descriptivo}

La población de estudio quedó conformada por 120 pacientes intervenidos de adenocarcinoma de próstata. Los datos descriptivos, antecedentes y resultados anatomopatológicos de la muestra se recogen en la - Tabla 1. La edad media de nuestra población fue de 68,37 (DE 11) años y el valor promedio del PSA fue de $12,17 \mathrm{ng} / \mathrm{ml}$. Aunque no hay un nivel específico de PSA que sea normal o anormal, el valor de referencia estándar de PSA en hombres sanos es de hasta $4 \mathrm{ng} / \mathrm{ml}$. Los niveles de PSA entre 4 y $10 \mathrm{ng} / \mathrm{ml}$ cuando la Densidad de PSA (DPSA) es menor de $0,15 \mathrm{ng} / \mathrm{ml} / \mathrm{cc}$, se asocian a menor riesgo de cáncer. El análisis anatomopatológico de la pieza quirúrgica describió más frecuentemente un Gleason de 6 o por debajo de esa cifra y el volumen prostático medio fue de 42,04 cc. De las 120 intervenciones realizadas, 73 se realizaron por vía abierta (16 casos laparoscópicos precisaron conversión) y las restantes fueron laparoscópicas. Se realizó el diagnóstico de EAU-V postquirúrgica (siempre mediante cistoscopia) en 27 (22,5\%) de los pacientes intervenidos y el tratamiento más empleado fue la incisión endoscópica de cuello vesical, por encima de las dilataciones. El 72,5\% (87) de los drenajes se mantuvieron menos de 3 días. Por los drenajes abdominales tipo Jackson-Pratt (J-P) se obtuvo más de 500cc en 62 (51,7\%; volumen medio: $1374 \mathrm{cc}$ ) de los casos. La mayoría de las intervenciones fueron realizadas por el Cirujano 2 (68 casos; 56,7\%) y por el Cirujano 3 (30 casos; 25\%).

\section{Análisis Estadístico de los Factores Asociados a la Aparición de EAU-V Posquirúrgica}

En nuestro estudio existe una asociación estadísticamente significativa de EAU-V posquirúrgica con el débito del drenaje quirúrgico J-P postoperatorio ( $\mathrm{p}=0,027$; IC 95\%: -3720; -241,75) (-Fig. 1), con los días de drenaje J-P ( $\mathrm{p}=0,009$; IC 95\%: $-2,05 ;-0,3$ ) (-Fig. 2 ) y con la estancia hospitalaria media $\left(X^{2}=5,35 ; \mathrm{p}=0,021\right)$. La incidencia de EAUV también se relacionó con Hemoglobina postoperatoria $<11 \mathrm{~g} / \mathrm{dL}(\mathrm{p}=0,039$; IC $95 \%$ : 0,$03 ; 1,26$; valores normales 13,3-16,2 $\mathrm{g} / \mathrm{dL}) \quad \mathrm{y}$ el hematocrito postoperatorio ( $\mathrm{p}=0,09$; IC $95 \%$ : 0,$63 ; 4,43$ ), con el cirujano que realizó la prostatectomía $\left(X^{2}=14,98 ; \mathrm{p}=0,010\right)$, con la densidad del PSA ( $p=0,008$; IC 95\%: 0,03; 0,25; valor asociado a un menor riesgo de cáncer $<0,15 \mathrm{ng} / \mathrm{mL} / \mathrm{cc}$ ) y con el PSA preoperatorio ( $\mathrm{p}=0,018$; IC 95\%: 0,73; 7,62; valor asociado a un menor riesgo de cáncer de hasta $4 \mathrm{ng} / \mathrm{mL}$ ).

\section{Análisis de Supervivencia}

Analizamos la supervivencia según si la cirugía se realizó vía abierta (incluiremos las cirugías reconvertidas) o laparoscópica. Del total de los 120 pacientes obtuvimos 73 que se intervinieron vía abierta por 47 laparoscópicos. En la cirugía abierta se obtuvieron 16 eventos con un 78,1\% de
Tabla 1 Características descriptivas de la muestra

\begin{tabular}{|c|c|}
\hline Edad media (años) & $68,37(\mathrm{DE} 11)$ \\
\hline Fumadores (\%) & $43(35,8 \%)$ \\
\hline \multicolumn{2}{|l|}{ Comorbilidades (\%) } \\
\hline - Diabetes Mellitus & $25(20,8 \%)$ \\
\hline - HTA & $65(54,2 \%)$ \\
\hline Radioterapia previa & $7(5,8 \%)$ \\
\hline Antecedentes de resección transuretral & $8(6,7 \%)$ \\
\hline \multicolumn{2}{|l|}{ Presentación del tumor } \\
\hline - Síndrome prostático & $76(63,3 \%)$ \\
\hline - Ningún síntoma al diagnóstico & $39(32,5 \%)$ \\
\hline - Hematuria & $5(4,2 \%)$ \\
\hline Valor promedio del PSA & $12,17 \mathrm{ng} / \mathrm{mL}$ \\
\hline \multicolumn{2}{|l|}{ Análisis anatomopatológico de la pieza } \\
\hline - Gleason $\leq 6$ & $104(86,2 \%)$ \\
\hline - Volumen prostático $\leq 70 \mathrm{cc}$ & $113(94,2 \%)$ \\
\hline - Infiltración de la cápsula & $70(58,3 \%)$ \\
\hline - Afectación vesical & $12(10 \%)$ \\
\hline - Márgenes afectados & $8(23,3 \%)$ \\
\hline - Afectación de cuello vesical & $5(4,2 \%)$ \\
\hline \multicolumn{2}{|l|}{ Clasificación para el estadiaje tumoral (TNM) } \\
\hline - T3aNOMO & $54(45 \%)$ \\
\hline - T2aNOM0 & $31(25,8 \%)$ \\
\hline - T2cNOMO & $16(13,3 \%)$ \\
\hline \multicolumn{2}{|l|}{ Vía de abordaje quirúrgico } \\
\hline - Abierto & $73(60,8 \%)$ \\
\hline - Laparoscopia & $47(39,2 \%)$ \\
\hline Tipo de sutura $2 / 0$ reabsorbible & $65(54,2 \%)$ \\
\hline \multicolumn{2}{|l|}{ Tiempo de cirugía } \\
\hline - superior a 3 horas & $\begin{array}{l}101 \text { pacientes } \\
(84,2 \%)\end{array}$ \\
\hline - tiempo quirúrgico medio (minutos) & 258 (DE 59,12) \\
\hline Estancia media (días) & 8,32 (DE 2,3) \\
\hline Tiempo medio de seguimiento (meses) & $41,46(\mathrm{DE} 12,2)$ \\
\hline Complicaciones postoperatorias & $13(10.8 \%)$ \\
\hline Reintervenciones & $5(4,2 \%)$ \\
\hline Fístulas postquirúrgicas & $2(1,7 \%)$ \\
\hline Enfermedad residual & $21(17,5 \%)$ \\
\hline Recurrencia bioquímica tras prostatectomía & $37(30,8 \%)$ \\
\hline \multicolumn{2}{|l|}{ EAU-V postquirúrgica } \\
\hline Tratamiento en las EAU-V & $27(22,5 \%)$ \\
\hline - Incisión endoscópica de cuello vesical & $67(56 \%)$ \\
\hline - Dilataciones & 53 (44\%). \\
\hline Calibre de la sonda vesical de $20 \mathrm{Fr}$ & $72(60 \%)$ \\
\hline
\end{tabular}

censurados, mientras que mediante vía laparoscópica 11 eventos con un $77,5 \%$ de censurados. En la curva de supervivencia de ambos grupos (-Fig. 3) se puede observar como en el grupo de "cirugía abierta" se produjeron EAU-V antes que en el grupo de cirugía laparoscópica, no 


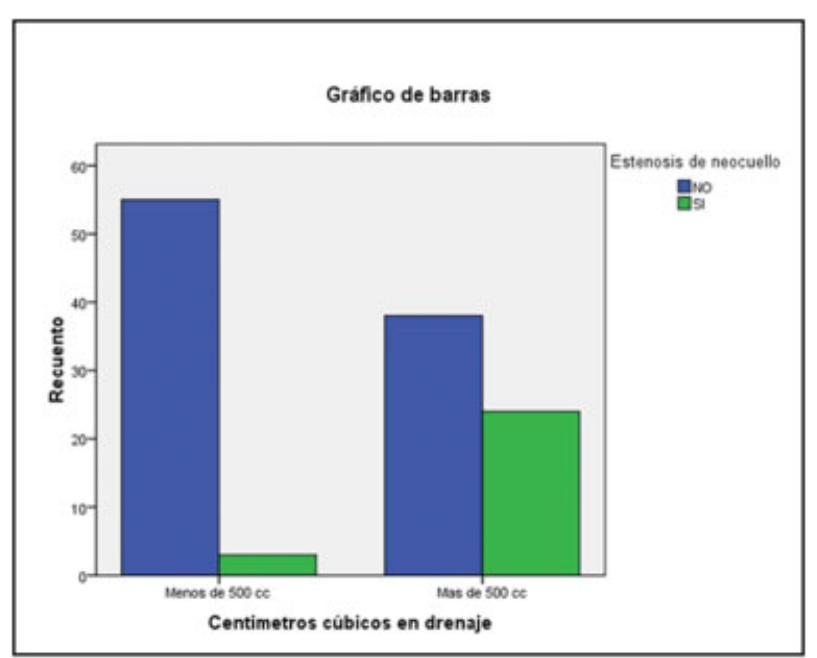

Fig. 1 Debito de drenaje como factor de riesgo para estenosis de neocuello.

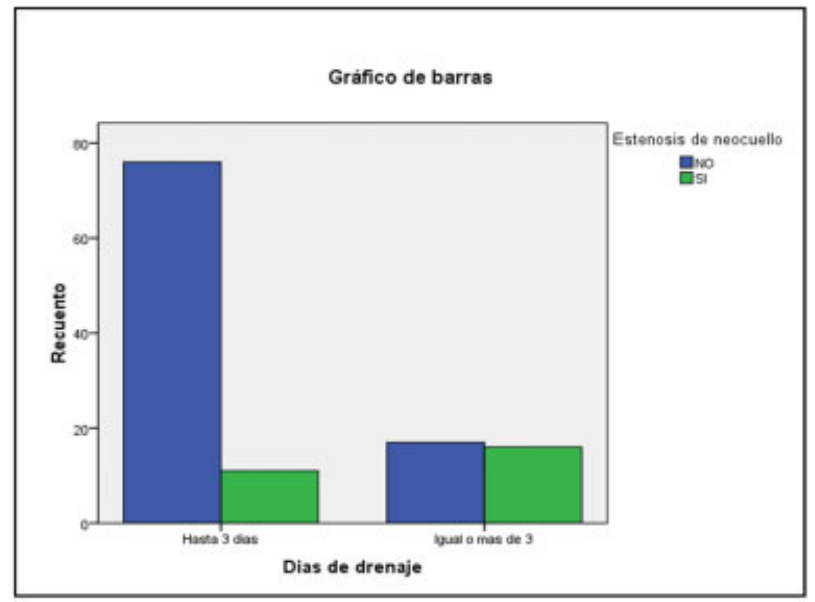

Fig. 2 Días de drenaje como factor de riesgo para estenosis de neocuello.

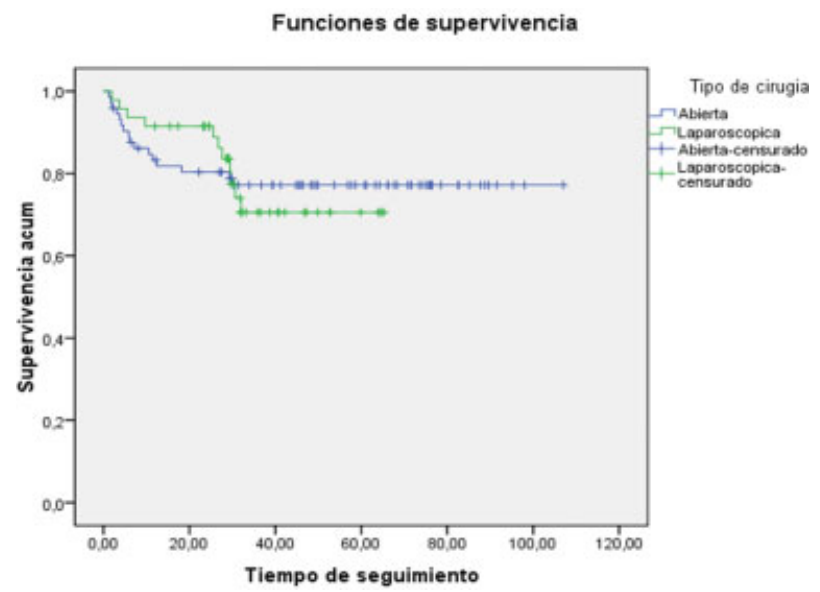

Fig. 3 Curva de supervivencia. Producción de estenosis a lo largo del tiempo. encontrándose diferencias estadísticamente significativas ( $\mathrm{p}=0,844$ ). Otro dato que aporta esa gráfica es que todas las estenosis se presentaron en los 3 primeros años tras la cirugía, tanto abierta como laparoscópica.

\section{Predicción del Riesgo de EAU-V Posquirúrgica en Pacientes a los que se ha Realizado Prostatectomía Radical por Adenocarcinoma de Próstata, Mediante un Modelo de Regresión de Riesgos Proporcionales de Cox} Obtenemos como resultado final que por mantener 3 o más días el drenaje J-P abdominal el riesgo de EAU-V se multiplicó por 3,21, lo que representa un factor de riesgo importante. Otro factor de riesgo fue tener más de 500 cc de débito por el drenaje quirúrgico J-P: multiplicó el riesgo de estenosis por 7,72.

\section{Discusión}

La incidencia de EAU-V postquirúrgica en nuestro estudio fue del 22,5\%, situándose entre los límites altos descritos en otros estudios ${ }^{1}$ en los que se reconoce una incidencia del 0,4-32\%. La variabilidad existente entre los distintos estudios publicados es debida, entre otros motivos, a diferencias en los criterios diagnósticos que hacen difícil conocer la incidencia real de esa complicación. También los criterios de selección de pacientes pueden provocar grandes diferencias con respecto a la incidencia, ya que algunos estudios incorporan pacientes intervenidos con una sola técnica quirúrgica o utilizando técnicas de las que no disponemos en nuestro hospital como la técnica laparoscópica asistida por robot.

Se han realizado múltiples estudios para determinar la incidencia de esa complicación así como los factores de riesgo para padecerla. Huang ${ }^{4}$ realizó un trabajo prospectivo incluyendo 708 pacientes intervenidos mediante prostatectomía abierta, concluyendo que la cantidad de sangrado y el calibre de sonda vesical se asociaban significativamente con la EAU-V. Erickson ${ }^{5}$ encontró asociación entre EAU-V y la experiencia del cirujano, así como con la preservación de nervios de la bandeleta neurovascular lateral a la próstata. Breyer ${ }^{6}$ realizó un estudio prospectivo sobre 988 pacientes comparando la incidencia de estenosis según se hubiera realizado prostatectomía radical abierta o por cirugía robótica, concluyendo que existía una baja incidencia, siendo algo mayor en el grupo intervenido mediante robot debido a factores técnicos de la cirugía. Otros autores, como Gotto, ${ }^{7}$ analizaron la incidencia de EAU-V tras tratamiento con técnicas no quirúrgicas como radioterapia o braquiterapia. Numerosos investigadores han destacado la mayor incidencia tras la utilización de clips metálicos intraoperatorios. ${ }^{8,9}$

La causa de EAU-V es probablemente multifactorial, desconociéndose actualmente el mecanismo fundamental de producción. En nuestro estudio existe una asociación estadísticamente significativa con los días de drenaje quirúrgico J-P postoperatorio, con el débito de ese drenaje $\mathrm{J}-\mathrm{P}$, con la estancia hospitalaria, con la hemoglobina y el hematocrito postoperatorio, con el cirujano que realiza la prostatectomía, con la densidad del PSA y con el PSA preoperatorio. En cuanto a los días de drenaje quirúrgico J-P 
parece lógico pensar que a más días, más extravasación urinaria se está produciendo y con ello mayor probabilidad de que se provoque una EAU-V. También se podría asociar a un sangrado postoperatorio abundante. Al comparar ese resultado con el obtenido por otros estudios se observa que la mayoría de ellos no estudian esa variable o bien se limitan a retirar el drenaje abdominal J-P pasado un número fijado de días. ${ }^{1}$

A pesar de no recoger la variable anterior, sí que hay descritos en la literatura trabajos que estudian la asociación de EAU-V y el recuento del material recogido por el drenaje quirúrgico. En ese caso, el método de producción de la estenosis sería el mismo. Uno de esos trabajos es el de Hanson ${ }^{10}$ donde se estudia la asociación entre la cantidad de material recogido por el drenaje abdominal y la incidencia de EAU-V, concluyendo que no existe tal asociación. En ese trabajo, las intervenciones son realizadas por prostatectomía abierta y mediante laparoscopia con robot, a diferencia del nuestro en el que las intervenciones fueron realizadas mediante prostatectomía abierta y laparoscópica. Además, los grupos que forma dependiendo de la cantidad obtenida, tienen valores diferentes a los que nosotros elegimos para nuestro estudio.

Respecto al tiempo de estancia hospitalaria, tampoco hemos encontrado en la literatura ningún artículo en el que esa variable haya dado una asociación significativamente estadística con la EAU-V postquirúrgica.

También hay trabajos ${ }^{3,4}$ que nos muestran que hay un mayor riesgo de estenosis según el cirujano que realice la intervención. En nuestro caso ocurriría lo mismo, observándose además que la mayoría de las intervenciones son realizadas por los mismos dos cirujanos.

Respecto a la pérdida sanguínea o al calibre de la sonda urinaria, también existen trabajos ${ }^{2}$ que encuentran un mayor riesgo de EAU-V dependiente de esos parámetros. En nuestro estudio, la pérdida sanguínea fue medida por las variables hemoglobina y hematocrito postoperatorios, observándose una relación estadísticamente significativa.

Sin embargo, el calibre de la sonda urinaria de nuestros pacientes fue poco variable e insuficiente para poder observar diferencias estadísticamente significativas. No parece haber relación entre el calibre de la sonda y la aparición de EAU-V.

En cuanto a la densidad del PSA y el PSA preoperatorio apreciamos como en nuestro estudio mostraron una asociación estadísticamente significativa con la EAU-V. No obstante, no damos mucha importancia a ese resultado ya que no pensamos que exista una relación clínicamente significativa.

El resto de variables del estudio no obtuvieron resultados estadísticamente significativos, en parte por no disponer de un tamaño muestral mayor: en el caso de las variables dependientes del tipo de sutura los datos coinciden con los de otros estudios ${ }^{11}$ en los que no se demuestra un mayor riesgo de sufrir EAU-V según el tipo de sutura utilizada o la cantidad de puntadas que se realicen.

En cuanto a la variable radioterapia previa, existen trabajos ${ }^{7}$ que demuestran un mayor riesgo de sufrir una EAU-V en los pacientes sometidos previamente a radioterapia.
Otro parámetro que en nuestro caso no ha dado significación estadística aunque sí en otros trabajos, ${ }^{12}$ es el tiempo de sondaje vesical. Bien es cierto que no hemos utilizado la cistografía para comprobar si existe extravasación a través de la anastomosis, y por lo tanto no es comparable.

Otros estudios incorporan variables como la utilización de clips $^{8,9}$ o la conservación de las bandeletas neurovasculares. ${ }^{3}$ En nuestro caso, eso fue imposible por no estar esos datos recogidos en la historia clínica.

En cuanto a la curva de supervivencia de los dos grupos (prostatectomía radical abierta vs laparoscópica ${ }^{13}$ ), se observó como en el grupo de "cirugía abierta" se producen EAU-V antes que en el grupo de "cirugía laparoscópica", no encontrándose diferencias estadísticamente significativas. Otro dato que aporta esa gráfica es que todas las estenosis se producen en los 3 primeros años tras la cirugía, tanto abierta como laparoscópica. La mayoría de los estudios que han aparecido en los últimos años comparan la supervivencia con el grupo de intervenidos con técnica laparoscópica asistida por robot ${ }^{6,14}$ por lo que nuestros resultados no son comparables al no disponer de esa tecnología. El que en nuestro caso no se hayan encontrado diferencias de supervivencia entre las dos técnicas quirúrgicas, puede ser debido a que en las primeras cirugías laparoscópicas realizadas por el Servicio, evitamos pacientes complejos, provocándose un posible sesgo de selección.

En lo referente al riesgo de estenosis, observamos en nuestro estudio como aquellos pacientes que tras ser intervenidos de prostatectomía radical, ya sea abierta o laparoscópica, van a presentar más riesgo de EAU-V en caso de presentar un debito del drenaje Jackson-Pratt mayor de 500cc o si el drenaje Jackson-Pratt no se puede retirar (por alto débito) antes del tercer día postoperatorio. Ese dato puede ayudarnos a la hora de formular un tipo de anamnesis distinto, centrado en la clínica provocada por la EAU-V, para aquellos pacientes que en su postoperatorio presentan un debito importante o un periodo de drenaje quirúrgico elevado.

Parece claro que deben existir otros factores no considerados en este estudio que pudieran añadirse a ese modelo predictivo y de así ayudarnos a conocer o sospechar qué pacientes pueden sufrir en el futuro una EAU-V.

Como en todos los estudios retrospectivos tenemos severas limitaciones. Aquellas variables no reflejadas en la historia del paciente se han perdido. Las asociaciones observadas aportan información que siempre debe corroborarse con otros estudios y es la observación repetida de resultados consistentes lo que sustenta la evidencia.

\section{Conclusiones}

La incidencia de EAU-V tras prostatectomía radical en nuestro Servicio es alta pero se acerca a la publicada por la mayoría de autores. $^{15,16}$ Se ha encontrado un mayor riesgo de padecer EAU-V postprostatectomía en aquellos pacientes en los que: a) se obtuvo por el drenaje quirúrgico tipo Jackson-Pratt 500cc o más centímetros cúbicos, b) el drenaje J-P no se pudo retirar 
por alto débito antes del tercer día postoperatorio, c) tuvieron una estancia hospitalaria de 8 o más días, d) tuvieron una hemoglobina o un hematocrito postoperatorio bajos, e) fueron intervenidos por el cirujano 1 o 5 , f) tuvieron un PSA preoperatorio bajo y g) tuvieron una densidad de PSA elevada.

Todas las estenosis se produjeron en los 3 primeros años tras la cirugía, tanto en cirugía abierta como laparoscópica, sin encontrarse diferencias estadísticamente significativas entre ambas técnicas.

\section{Responsabilidades Éticas}

Protección de personas y animales. Los autores declaran que los procedimientos seguidos se conformaron a las normas éticas del comité de experimentación humana responsable y de acuerdo con la Asociación Médica Mundial y la Declaración de Helsinki.

Confidencialidad de los datos. Los autores declaran que han seguido los protocolos de su centro de trabajo sobre la publicación de datos de pacientes.

Derecho a la privacidad y consentimiento informado. Los autores declaran que en este artículo no aparecen datos de pacientes.

Conflicto de Intereses

No existe conflicto de intereses para ninguno de los autores.

\section{Referencias}

1 Besarani D, Amoroso P, Kirby R. Bladder neck contracture after radical retropubic prostatectomy. BJU Int 2004;94(09):1245-1247

2 Elliott SP, Meng MV, Elkin EP, McAninch JW, Duchane J, Carroll PR; CaPSURE Investigators. Incidence of urethral stricture after primary treatment for prostate cancer: data From CaPSURE. J Urol 2007;178(02):529-534, discussion 534

3 Kovell RC, Terlecki RP. Management Strategies for PostProstatectomy Bladder Neck Contractures. Curr Urol Rep 2015; 16(09):65. DOI: 10.1007/s11934-015-0536-4
4 Huang G, Lepor H. Factors predisposing to the development of anastomotic strictures in a single-surgeon series of radical retropubic prostatectomies. BJU Int 2006;97(02):255-258

5 Erickson BA, Meeks JJ, Roehl KA, González CM, Catalona WJ. Bladder neck contracture after retropubic radical prostatectomy: incidence and risk factors from a large single-surgeon experience. BJU Int 2009;104(11):1615-1619

6 Breyer BN, Davis CB, Cowan JE, Kane CJ, Carroll PR. Incidence of bladder neck contracture after robot-assisted laparoscopic and open radical prostatectomy. BJU Int 2010;106(11):1734-1738

7 Gotto GT, Yunis LH, Vora K, Eastham JA, Scardino PT, Rabbani F. Impact of prior prostate radiation on complications after radical prostatectomy. J Urol 2010;184(01):136-142

8 Yi JS, Kwak C, Kim HH, Ku JH. Surgical clip-related complications after radical prostatectomy. Korean J Urol 2010;51(10):683-687

9 Blumenthal KB, Sutherland DE, Wagner KR, Frazier HA, Engel JD. Bladder neck contractures related to the use of Hem-o-lok clips in robot-assisted laparoscopic radical prostatectomy. Urology 2008; 72(01):158-161

10 Hanson GR, Odom E, Borden LS Jr, Neil N, Corman JM. Postoperative drain output as a predictor of bladder neck contracture following radical prostatectomy. Int Urol Nephrol 2008;40(02): 351-354

11 Mazaris EM, Chatzidarellis E, Varkarakis IM, Dellis A, Deliveliotis C. Reducing the number of sutures for vesicourethral anastomosis in radical retropubic prostatectomy. Int Braz J Urol 2009;35(02): 158-163

12 Tiguert R, Rigaud J, Fradet Y. Safety and outcome of early catheter removal after radical retropubic prostatectomy. Urology 2004;63 (03):513-517

13 Raventós Busquets CX, Gómez Lanza E, Cecchini Rosell L, et al. Prostatectomia radical laparoscópica versus abierta. Actas Urol Esp 2007;31(02):141-145

14 Martínez-Salamanca JI, Allona Almagro A. Prostatectomia radical abierta, laparoscópica y robótica: En busca de un nuevo gold standard? Actas Urol Esp 2007;31(04):316-327

15 Fernández Ortiz D, Villamil W, Cristalo C, et al. Abordaje quirúrgico de la estenosis de la anastomosis uretrovesical post prostatectomía radical. Rev Arg de Urol 2014;79(01):11-18

16 Muñoz D, Vicens A, García-Montes F. Estenosis de la anastomosis vesicouretral tras prostatectomía radical y radioterapia postoperatoria. Actas Urol Esp 2011;35(05):277-281 